\title{
COX-2 inhibition with rofecoxib does not increase intestinal permeability in healthy subjects: a double blind crossover study comparing rofecoxib with placebo and indomethacin
}

\author{
G Sigthorsson, R Crane, T Simon, M Hoover, H Quan, J Bolognese, I Bjarnason
}

\begin{abstract}
Background-Acute and chronic use of non-steroidal anti-inflammatory drugs can increase intestinal permeability. Rofecoxib, which selectively inhibits cyclooxygenase 2 (COX-2), is a novel antiinflammatory drug with the potential to produce minimal gastrointestinal toxic effects while retaining clinical efficacy.

Aims-To assess the potential for rofecoxib to affect the intestine adversely, in comparison with placebo and indomethacin.

Subjects-Thirty nine healthy subjects (aged 24-30 years).

Method-We performed a four period crossover trial to assess intestinal permeability before and after seven days of treatment. Permeability was measured by the urinary ratio of chromium-51 labelled ethylene diamine tetraacetate $\left({ }^{51} \mathrm{CrEDTA}\right) / \mathrm{L}-\mathrm{rhamnose}$ (five hour collection).
\end{abstract}

Results-Indomethacin $50 \mathrm{mg}$ three times daily produced greater increases in intestinal permeability compared with placebo or rofecoxib (25 or $50 \mathrm{mg}) \quad(p \leqslant 0.001)$; rofecoxib was not significantly different from placebo. Mean day 7 to baseline ratios $(95 \%$ confidence intervals) for ${ }^{51} \mathrm{CrEDTA} / \mathrm{L}-$ rhamnose were $0.97 \quad \mathbf{0 . 8 2}$, 1.16), 0.80 (0.68, 0.95), 0.98 (0.82, 1.17), and $1.53(1.27,1.85)$ for placebo, rofecoxib $25 \mathrm{mg}$, rofecoxib $50 \mathrm{mg}$, and indomethacin groups, respectively. Rofecoxib was generally well tolerated.

Conclusion-In this study, treatment for one week with indomethacin $50 \mathrm{mg}$ three times daily significantly increased intestinal permeability compared with placebo, while treatment with rofecoxib $25 \mathrm{mg}$ or 50 mg daily did not. The absence of a significant effect of rofecoxib on intestinal permeability at doses at least twice those recommended to treat osteoarthritis was consistent with other studies that have demonstrated little or no injury to the gastrointestinal mucosa associated with rofecoxib therapy.

(Gut 2000;47:527-532)

Keywords: rofecoxib; COX-2 inhibitor; indomethacin; non-steroidal anti-inflammatory drugs; intestinal permeability; osteoarthritis
Non-steroidal anti-inflammatory drugs (NSAIDs) are effective in treating a variety of inflammatory diseases but frequently produce untoward reactions, particularly in the gastrointestinal tract. ${ }^{1}$ While gastropathy (for example, upper gastrointestinal bleeding, ulcer, and perforation) represents the most common serious and life threatening complication of NSAID therapy, damage to the intestinal mucosa also occurs during long term NSAID treatment. Indeed, as many as $70 \%$ of patients receiving long term NSAID therapy develop asymptomatic small intestinal inflammation, which may subsequently lead to blood and protein loss, ileal dysfunction, and the development of circumferential, diaphragmatic small intestinal strictures..$^{2-6}$

A prerequisite for the development of NSAID associated small intestinal inflammation is believed to involve an increase in intestinal permeability, as evidenced by enhanced permeation of chromium-51 labelled ethylene diamine tetraacetate $\left({ }^{51} \mathrm{CrEDTA}\right) .{ }^{7}$ Increased intestinal permeability induced by NSAIDs may expose the mucosa to endogenous and exogenous luminal toxins, ultimately allowing bacterial invasion of the intestinal mucosa and development of a local inflammatory response. $^{89}$

The propensity of NSAIDs to increase intestinal permeability may be linked to the ability to deplete endogenous intestinal mucosal prostaglandins and/or interfere with mitochondrial energy metabolism. ${ }^{10}$ The latter assertion is supported by animal experiments ${ }^{11}$ while the former is supported by the findings that the magnitude of enhanced intestinal permeability produced by NSAIDs is quantitatively related to their potency in inhibiting cyclooxygenase and that changes in intestinal permeability induced by NSAIDs can be attenuated by coadministration of the prostaglandin analogue misoprostol in humans. ${ }^{12} 13$

Cyclooxygenase 1 (COX-1) and 2 (COX-2) are key enzymes involved in prostaglandin synthesis in humans. ${ }^{1}$ COX-1, a constitutively expressed enzyme present in most cells and tissues throughout the body, is responsible for generating prostaglandins involved in various

Abbreviations used in this paper: NSAIDs, non-steroidal anti-inflammatory drugs; ${ }^{51} \mathrm{CrEDTA}$, chromium-51 labelled ethylene diamine tetraacetate; COX-1, cyclooxygenase 1 ; COX-2, cyclooxygenase 2 ; $\beta$-HCG, $\beta$ human chorionic gonadotropin. 
physiological functions, including gastrointestinal mucosal protection. ${ }^{14}$ In contrast, the COX-2 isoform is rapidly induced at sites of inflammation and generates prostaglandins that mediate local inflammation and pain. ${ }^{15}$ The deleterious effects of NSAIDs in the gastrointestinal tract are believed to occur primarily as a result of blockade of COX-1 activity, whereas the anti-inflammatory effects of NSAIDs are mediated through inhibition of COX-2. ${ }^{16}$ Recently, compounds have been developed that are highly selective COX-2 inhibitors with little or no effect on COX-1 (coxibs, such as rofecoxib). Rofecoxib has been shown to be clinically effective in treating osteoarthritis at doses of $12.5-25 \mathrm{mg}$ daily. ${ }^{17}{ }^{18}$ Rofecoxib has also been shown to inhibit COX-2 selectively in vitro ${ }^{19}{ }^{20}$; in human whole blood, it did not inhibit thromboxane $\mathrm{B}_{2}$ (a measure of COX-1 activity) at doses up to $1000 \mathrm{mg} /$ day. ${ }^{21}$ Since rofecoxib is non-acidic and has no significant effect on prostaglandin synthesis in human gastric biopsies at therapeutic doses, ${ }^{22}$ this anti-inflammatory agent may have greatly reduced potential to produce gastrointestinal toxic effects. ${ }^{2324}$

To gain insight into the potential for rofecoxib to affect the intestine, we compared the effects of rofecoxib, indomethacin (a non-selective inhibitor of cyclooxygenase), and placebo on the permeability of the intestine after seven days of treatment in healthy subjects in a double blind crossover study.

\section{Subjects and methods}

This double blind, four period crossover study compared the effects of seven days of treatment with rofecoxib (25 or $50 \mathrm{mg}$ once daily), indomethacin (50 $\mathrm{mg}$ three times daily), or placebo on intestinal permeability, as assessed by differential five hour urinary excretion of ${ }^{51} \mathrm{CrEDTA} / \mathrm{L}-$ rhamnose, in a total of 39 healthy subjects. Included in the trial were male and female subjects, aged 18-39 years, who were within $20 \%$ of ideal body weight based on the Metropolitan Life Height and Weight Tables. Female subjects of childbearing potential were required to have a negative pregnancy test, as assessed by measuring serum $\beta$ human chorionic gonadotropin ( $\beta-\mathrm{HCG}$ ) levels, and to use double barrier birth control methods beginning one week before and continuing for at least two weeks after the study. Subjects were excluded if they had a history of peptic ulcers, gastro-oesophageal reflux disease, inflammatory bowel disease, irritable bowel syndrome, pancreatic or biliary disorders, or other significant gastrointestinal disease, a history of gastrointestinal surgery (other than appendicectomy or inguinal hernia repair), or a history of frequent use ( $>2$ twice a month) of antacids, $\mathrm{H}_{2}$ receptor antagonists, or proton pump inhibitors. Subjects were also excluded if they had participated in an investigational study within four months prior to study entry, had a positive faecal occult blood screening test, significant abnormalities on haematological or biochemical screening, or had known significant medical conditions such as heart disease, blood dyscrasias or coagulopathy, neurological disease, hepatic/renal dysfunction, active malignant disease, hypertension, or diabetes. In addition, subjects were excluded if they were drug or alcohol dependent or allergic or intolerant to diclofenac, indomethacin, aspirin, or other non-selective inhibitors of cyclooxygenase. The use of prescription or nonprescription preparations containing aspirin or NSAIDs (including over the counter drugs) was prohibited within two weeks prior to study entry and during the study. The study was approved by the institutional review board of King's College School of Medicine and all subjects were required to give written informed consent before participating in the study.

\section{STUDY DESIGN}

At an initial screening visit, subjects underwent a complete medical history, routine laboratory evaluation, faecal occult blood testing, and pregnancy testing (serum $\beta$-HCG levels) for female subjects. Subjects were asked to abstain completely from alcoholic beverages, spicy foods, and smoking for seven days before and during the study and were instructed to discontinue any prior prescription and nonprescription medications (including over the counter preparations) containing any NSAIDs and/or antisecretory or cytoprotective drug therapies.

Approximately one week after screening, eligible subjects underwent a baseline evaluation of intestinal permeability (see below). A total of 39 subjects were randomised to receive one of four treatment regimens for seven days: placebo; rofecoxib $25 \mathrm{mg}$ once daily in the morning; rofecoxib $50 \mathrm{mg}$ once daily in the morning; or indomethacin $50 \mathrm{mg}$ (two $25 \mathrm{mg}$ tablets) three times daily. Each subject received all four seven day treatment regimens in a predefined balanced sequence and each treatment regimen was separated by a washout period of 7-10 days. Study medication was dispensed in individually marked boxes (three boxes per treatment period) containing a seven day supply of study drugs. During the study, subjects completed diaries to record compliance with study medication. After the initial dose of study medication, subjects were reminded by electronic pager to take subsequent doses throughout the entire treatment period. Subjects who discontinued prior to completion of the protocol were replaced with subjects assigned to the same treatment order to preserve statistical balance.

\section{MEASUREMENT OF INTESTINAL PERMEABILITY}

Intestinal permeability was determined by measuring urinary recovery of ${ }^{51}$ CrEDTA (a marker of intercellular permeation) and L-rhamnose (a marker of non-carrier mediated transcellular permeation). ${ }^{8}$ The urinary excretion ratio of ${ }^{51} \mathrm{CrEDTA} / \mathrm{L}-$ rhamnose was chosen as the primary index of intestinal permeability because the differential excretion ratio of these agents is largely unaffected by premucosal and postmucosal factors. ${ }^{25}$ We chose ${ }^{51}$ CrEDTA rather than lactulose as a paracellular marker in this study to maximise the sensitivity of the small intestinal permeability test. 
Previous studies have shown that differential urinary excretion of ${ }^{51} \mathrm{CrEDTA} / \mathrm{L}-\mathrm{rhamnose}$ is more sensitive than lactulose/L-rhamnose (when administered as an iso-osmolar test solution) in patients with coeliac disease ${ }^{26}$ or taking NSAIDs. ${ }^{8}$

Intestinal permeability was measured before treatment in each of the four treatment periods and on day 7 at the conclusion of each treatment period. For each permeability measurement, fasting subjects drank a test solution $(100 \mathrm{ml})$ containing ${ }^{51}$ CrEDTA (30 $\mu \mathrm{Ci}$, Amersham International, Amersham, UK), L-rhamnose (1.0 g), and D-xylose $(0.5 \mathrm{~g})$ one hour after consuming the study medication (except at baseline when no study medication was taken). The radiation dose received from a $30 \mathrm{mCi}$ dose of ${ }^{51} \mathrm{CrEDTA}$ taken orally is less than 0.04 millisieverts (effective dose equivalent). D-Xylose $(0.5 \mathrm{~g})$ was included in the test solution as a marker of intestinal absorption, as some, ${ }^{27}{ }^{28}$ but not all, ${ }^{12}$ studies have suggested that NSAIDs impair absorption. Urine samples were subsequently collected over the next five hours, during which subjects continued fasting for the first two hours. Urine was collected in a bottle containing $1 \mathrm{ml}(10 \% \mathrm{w} / \mathrm{v})$ of sodium ethylmercurithiosalicylate (thiomersal, Sigma Chemical Co., St Louis, Missouri, USA) as a preservative. Urine volume was measured on completion of the test, and a $5 \mathrm{ml}$ sample was removed for measurement of radioactivity using an LKB 1281 gamma counter. Urinary concentrations of L-rhamnose and D-xylose were determined using a modified thin layer chromatographic technique which involved scanning densitometry with an arabinose internal standard to overcome errors of application, as described previously. ${ }^{29}$ The percentage of the ${ }^{51} \mathrm{CrEDTA}$ and L-rhamnose dose was determined in each urine sample, from which the ratio of the percentage excreted ${ }^{51} \mathrm{CrEDTA} /$ L-rhamnose was calculated.

TOLERABILITY ASSESSMENTS

Adverse experiences were monitored throughout the study and were defined as any unfavourable or unintended change in structure, function, or chemistry of the body, or a worsening of a pre-existing condition. Investigators evaluated all clinical adverse experiences in terms of intensity (mild, moderate, or severe), duration, seriousness, outcome, and relation to test drugs.

STATISTICAL ANALYSIS

Data obtained from a previously published study $^{8}$ indicated that the ${ }^{51} \mathrm{CrEDTA} /$ L-rhamnose ratio is log normally distributed. Therefore, between treatment comparisons were made by computing the geometric mean ratio of ${ }^{51} \mathrm{CrEDTA} / \mathrm{L}-$ rhamnose values. Based on pre- and post-treatment data from this study, ${ }^{8}$ a limit for comparability to placebo was prespecified; rofecoxib would be considered equivalent to placebo if the between treatment ratio of the ${ }^{51} \mathrm{CrEDTA} / \mathrm{L}-$ rhamnose values was $<1.37$. The sample size was chosen to provide $95 \%$ power to prove that the upper limit of a 95\% two sided confidence interval was below this value. The primary statistical analysis was based on a per protocol assessment of data. However, if a subject completed periods 1 and 2 and then discontinued the study, the subject was still included in the analysis, even though the subject failed to complete the entire study.

Analysis of covariance suitable for a four period crossover design (with treatment, period, subject, carryover, and log baseline as covariate factors) was used to analyse differences in the $\log$ of ${ }^{51} \mathrm{CrEDTA} / \mathrm{L}-$ rhamnose ratio on day 7 compared with baseline values and to assess the statistical significance of differences in the $\log$ of ${ }^{51} \mathrm{CrEDTA} / \mathrm{L}$-rhamnose ratio among the four study groups.

\section{Results}

STUDY POPULATION AT ENTRY

A total of 39 subjects were enrolled in the study. Mean age was 28 years (range 18-39 years) and $54 \%$ were male. Subjects were assigned to one of four treatment sequence groups and received, in a predefined balanced sequence (for first order carryover effect), the four study drugs (placebo, rofecoxib $25 \mathrm{mg}$, rofecoxib $50 \mathrm{mg}$, and indomethacin $150 \mathrm{mg}$ ). The treatment sequence groups were well matched with respect to baseline characteristics, including age, sex, race, caffeine use, secondary diagnoses, prior medication use, and baseline intestinal permeability. All subjects had a negative faecal occult blood test. Nine subjects violated the study protocol by consuming alcohol and were therefore excluded from the per protocol analysis (alcohol may affect intestinal permeability). In addition, isolated visits from five other subjects were also excluded because of protocol violations; all decisions regarding protocol violations were made before unblinding the treatment code. Three subjects discontinued prior to study completion of all four treatment groups; one subject developed an adverse experience during treatment. At the time of discontinuation, one subject was taking placebo, and the other two subjects discontinued during a washout period following rofecoxib $50 \mathrm{mg}$. A comparison of the effects of the four study drugs on intestinal permeability (per protocol) was made on the following numbers of patients: 29 in the placebo group, 29 in the rofecoxib $25 \mathrm{mg}$ group, 27 in the rofecoxib $50 \mathrm{mg}$ group, and 26 in the indomethacin $150 \mathrm{mg}$ group.

EFFECT ON INTESTINAL PERMEABILITY

Pretreatment differential urinary ratios of ${ }^{51} \mathrm{CrEDTA} / \mathrm{L}$-rhamnose were comparable across the four treatment regimens, with mean values of $0.036,0.036,0.035$, and 0.038 for placebo, rofecoxib $25 \mathrm{mg}$, rofecoxib $50 \mathrm{mg}$, and indomethacin $150 \mathrm{mg}$, respectively (fig 1 ). Following seven days of administration of indomethacin, the permeability of the intestine increased significantly, as indicated by an increase in the ratio of ${ }^{51} \mathrm{CrEDTA} / \mathrm{L}$-rhamnose from baseline values of 0.038 to 0.055 on day 7 $(p<0.05)$. In contrast, the permeability of the intestine following seven days of administration of placebo, rofecoxib $25 \mathrm{mg}$, or rofecoxib 50 mg was not significantly different from baseline 


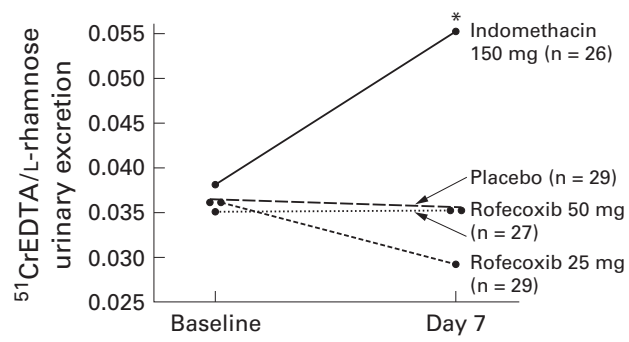

Figure 1 Five hour geometric mean urinary excretion ratios for ${ }^{51} \mathrm{CrEDTA} / \mathrm{L}$-rhamnose at baseline and on day 7 for subjects who received placebo, rofecoxib $25 \mathrm{mg}$, rofecoxib $50 \mathrm{mg}$, and indomethacin $150 \mathrm{mg}$ (per protocol analysis). Indomethacin $150 \mathrm{mg}$ significantly increased the ${ }^{51} \mathrm{CrEDTA} / \mathrm{L}$-rhamnose ratio compared with baseline values, whereas placebo, rofecoxib $25 \mathrm{mg}$, and rofecoxib 50 $m g$ did not. ${ }^{*} p<0.05$ versus baseline values

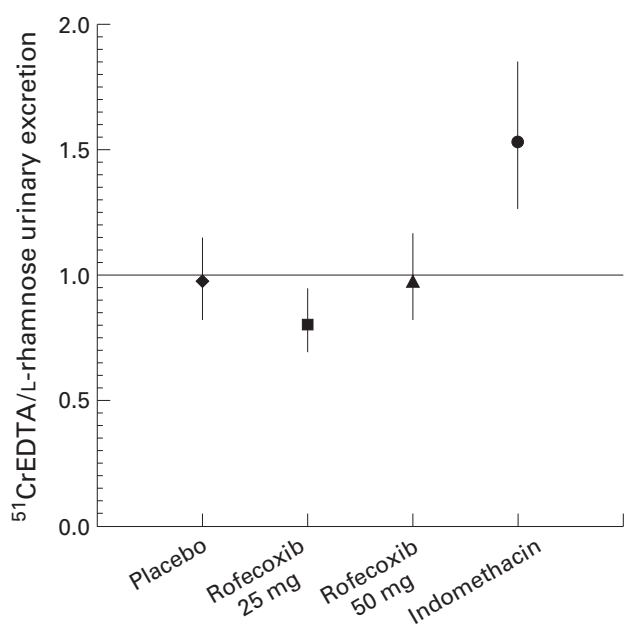

Figure 2 Day 7 to baseline geometric mean ratios (with $95 \%$ confidence intervals) for five hour urinary excretion of ${ }^{51} \mathrm{CrEDTA} / \mathrm{L}$-rhamnose in subjects who received placebo, rofecoxib $25 \mathrm{mg}$, rofecoxib $50 \mathrm{mg}$, and indomethacin 150 $m g$ (per protocol analysis).

values (fig 1). As illustrated in fig 2, day 7 to baseline ratios for ${ }^{51} \mathrm{CrEDTA} / \mathrm{L}-\mathrm{rhamnose}$ averaged $0.97,0.80,0.98$, and 1.53 for placebo, rofecoxib $25 \mathrm{mg}$, rofecoxib $50 \mathrm{mg}$, and indomethacin $150 \mathrm{mg}$ groups, respectively. Analysis of intent to treat data, which included all patients enrolled in the study, revealed similar findings to those seen with the per protocol analysis.

Comparison of day 7 to baseline ratios for ${ }^{51} \mathrm{CrEDTA} / \mathrm{L}-\mathrm{rhamnnose}$ revealed no significant differences between placebo and either the rofecoxib $25 \mathrm{mg}$ or rofecoxib $50 \mathrm{mg}$ groups (table 1); the upper limit on the $95 \%$ confidence interval was $<1.37$, the prespecified definition of clinical equivalence. In contrast, day 7 to baseline ratio for ${ }^{51} \mathrm{CrEDTA} /$

Table 1 Between group comparison of day 7 to baseline ratios for five hour urinary excretion of ${ }^{51} \mathrm{CrEDTA} / \mathrm{L}$-rhamnose (per protocol analysis)

\begin{tabular}{llll}
\hline & $\begin{array}{c}\text { Day 7/baseline ratio for } \\
\end{array}$ & & \\
& ${ }^{51}$ CrEDTA/L-rhamnose & $95 \%$ CI & p Value \\
\hline Rofecoxib 25 mg $v$ placebo & 0.82 & $0.65,1.05^{\star}$ & $\mathrm{NS}$ \\
Rofecoxib 50 mg $v$ placebo & 1.01 & $0.79,1.29^{\star}$ & $\mathrm{NS}$ \\
Indomethacin 150 mg $v$ placebo & 1.58 & $1.22,2.04$ & 0.001 \\
Indomethacin 150 mg v rofecoxib 25 mg & 1.91 & $1.47,2.48$ & $<0.001$ \\
Indomethacin 150 mg v rofecoxib 50 mg & 1.56 & $1.21,2.01$ & 0.001 \\
\hline
\end{tabular}

*Upper limit did not exceed 1.37 (that is, the upper 95\% confidence interval for the rofecoxib $v$ placebo ${ }^{51} \mathrm{CrEDTA} / \mathrm{L}-$ rhamnose ratios).

NS, not significant.
L-rhamnnose was significantly higher in subjects who received indomethacin compared with either the placebo or rofecoxib groups $(\mathrm{p} \leqslant 0.001)$.

Absorption of D-xylose was not significantly different between the four treatment groups $(p=0.382)$. Day 7 to baseline mean ratios for urinary excretion of $\mathrm{D}$-xylose were $0.95,1.05$, 0.99 , and 0.87 for placebo, rofecoxib $25 \mathrm{mg}$, rofecoxib $50 \mathrm{mg}$, and indomethacin $150 \mathrm{mg}$ groups, respectively. None of these ratios was significantly different from 1.0 (data not shown).

\section{SAFETY}

No serious clinical or laboratory adverse experiences occurred during the study. Non-serious adverse effects were reported more often in subjects receiving indomethacin $150 \mathrm{mg}$ $(83.3 \%)$ than in subjects who received placebo, rofecoxib $25 \mathrm{mg}$, or rofecoxib $50 \mathrm{mg}(53.8 \%$, $64.1 \%$, and $60.5 \%$, respectively). Overall, the incidence of clinical adverse experiences was similar across treatment groups except for dizziness and headache which were reported more often in indomethacin treated subjects than in placebo or rofecoxib treated subjects. Analysis of the occurrence of predefined NSAID-type gastrointestinal symptoms (acid reflux, dyspepsia, epigastric discomfort, heartburn, nausea, and vomiting) showed that overall, gastrointestinal symptoms occurred most frequently with indomethacin treatment $(30.6 \%)$, had a similar frequency in the rofecoxib $50 \mathrm{mg}$ and placebo groups $(18.4 \%$ and $12.8 \%$, respectively), and were least frequent in the rofecoxib $25 \mathrm{mg}$ group ( $5.1 \%)$. One subject discontinued the study due to an adverse event which occurred during treatment with placebo (angioedema). Two subjects discontinued because of adverse events during washout periods (one due to pyelonephritis, one due to upper respiratory infection with fever, abdominal pain, and diarrhoea). None of the reported adverse experiences necessitated unblinding of treatment.

\section{Discussion}

This double blind, four period crossover study in healthy subjects showed that intestinal permeability (as assessed by five hour ${ }^{51} \mathrm{CrEDTA} / \mathrm{L}$-rhamnnose urinary excretion ratio) after seven days of treatment with the highly selective COX-2 inhibitor rofecoxib (25 $\mathrm{mg}$ or $50 \mathrm{mg}$ daily) was not significantly different from that observed in subjects receiving placebo. In contrast, seven day administration of indomethacin significantly increased intestinal permeability compared with both placebo and rofecoxib (25 or $50 \mathrm{mg}$ ); prespecified criteria for equivalence to placebo were satisfied. As increased intestinal permeability is considered to be a prerequisite for development of NSAID induced enteropathy, these findings suggest that rofecoxib may, in the long term, be superior to NSAIDs with respect to the development of intestinal complications typically associated with non-selective inhibitors of cyclooxygenase. 
The lack of effect of rofecoxib on intestinal permeability in this study is consistent with the findings of previous clinical studies showing little or no gastrointestinal toxicity associated with rofecoxib therapy. For example, a recent double blind study in healthy subjects demonstrated that gastrointestinal microbleeding (measured using ${ }^{51} \mathrm{Cr}$ labelled red blood cells) during rofecoxib therapy (25 or $50 \mathrm{mg}$ daily) was statistically equivalent to that seen during placebo treatment and was significantly less than in subjects treated with ibuprofen (2400 mg daily). ${ }^{24}$ Similarly, at doses at least twice those required for clinical efficacy in treating osteoarthritis, rofecoxib caused significantly less gastroduodenal ulceration detected by endoscopy than ibuprofen and was again comparable with placebo in terms of gastroduodenal toxicity. ${ }^{30}$ Even at daily doses as high as 250 $\mathrm{mg}$, corresponding to 20 times the dose demonstrated to relieve symptoms of osteoarthritis, rofecoxib produced markedly less endoscopic evidence of gastroduodenal injury than ibuprofen $800 \mathrm{mg}$ three times daily or aspirin $650 \mathrm{mg}$ four times daily and was similar to placebo. ${ }^{31}$ Collectively, these studies indicate that rofecoxib, which selectively inhibits COX-2, has the potential to combine antiinflammatory efficacy with an improved gastrointestinal safety profile.

NSAIDs have been shown previously to increase intestinal permeability. ${ }^{32}$ Indomethacin was chosen as the NSAID comparator for this study as it is the most widely used NSAID for this type of study, and it is known to disrupt the integrity of the intestinal mucosa rapidly and reversibly on discontinuation of drug intake. The mechanism of indomethacin induced increased intestinal permeability is controversial and may involve an effect on mitochondria, which is dependent on the drug being acidic and/or its effect in decreasing mucosal prostaglandins. ${ }^{13}{ }^{33}$ Whatever the precise mechanism, the data suggest that the NSAID induced increase in differential urinary excretion of $51 \mathrm{CrEDTA} / \mathrm{L}-$ rhamnose represents damage to intercellular junctions, a prerequisite for NSAID induced enteropathy. ${ }^{910}$ A further consideration is whether it is valid to extrapolate data from young healthy volunteers, who did not have a history of gastrointestinal disturbances, to the elderly population who may use rofecoxib. Although limited information from previous studies has not shown any difference between the prevalence or magnitude of increased intestinal permeability or the inflammatory changes due to NSAIDs among young and old patients or volunteers, ${ }^{34}$ longer term studies focused on elderly patients or those with specific risk factors may be useful in the future to define intestinal permeability more precisely in these subpopulations of NSAID users.

The dose of indomethacin used $(50 \mathrm{mg}$ three times daily) is frequently prescribed to treat anti-inflammatory conditions such as osteoarthritis and rheumatoid arthritis. The doses of rofecoxib chosen to assess the potential of this highly selective COX-2 inhibitor to increase intestinal permeability were $2-4$ times the doses that relieved the symptoms of osteoarthritis. ${ }^{17}$

In keeping with previous studies, indomethacin increased intestinal permeability significantly. However, rofecoxib had no effect on intestinal permeability. This is consistent with the non-acidic nature of rofecoxib and its metabolites as well as the fact that it does not affect intestinal prostanoid metabolism or levels in humans or in experimental animals at much higher doses.

Rofecoxib was generally well tolerated after seven days of treatment and was not associated with any serious adverse effects. The incidences of adverse clinical and laboratory events observed with rofecoxib $25 \mathrm{mg}$ or rofecoxib 50 $\mathrm{mg}$ doses were similar to those reported in the placebo group. The incidence of selected gastrointestinal adverse experiences commonly associated with NSAID therapy (for example, acid reflux, dyspepsia, epigastric discomfort, heartburn, nausea, and vomiting) was highest in the indomethacin group, similar between the rofecoxib $50 \mathrm{mg}$ and placebo groups, and lowest in the rofecoxib $25 \mathrm{mg}$ group.

The absence of a significant effect of rofecoxib on intestinal permeability, which is generally considered to represent a prerequisite step in the development of NSAID induced enteropathy, is consistent with previous studies showing little or no gastrointestinal injury associated with rofecoxib therapy. This study was funded by a grant from Merck \& Co., Inc., the
manufacturers of rofecoxib. T Simon, $M$ Hoover, H Quan, and manufacturers of rofecoxib. T Simon,

1 Cryer B. Nonsteroidal anti-inflammatory drugs and gastrontestinal disease. In: Feldman $M$, Scharschmidt BF, Sleisenger MH, eds. Sleisenger and Fordtran's gastrointestinal and liver disease, 6th edn. Philadelphia: Saunders, 1998:343-57.

2 Bjarnason I, Williams $\mathrm{P}$, So A, et al. Intestinal permeability and inflammation in rheumatoid arthritis: effects of non-steroidal anti-inflammatory drugs. Lancet 1984;2: 1171-4.

3 Segal AW, Isenberg DA, Hajirousou V, et al. Preliminary evidence for gut involvement in the pathogenesis of rheumadence for gut involvement in the pathogenesis
toid arthritis? Br 7 Rheumatol 1986;25:162-6.

4 Bjarnason I, Zanelli G, Smith T, et al. Nonsteroidal antiinflammatory drug-induced intestinal inflammation in inflammatory drug-induced intestinal

5 Bjarnason I, Zanelli G, Prouse P, et al. Blood and protein oss via small-intestinal inflammation induced by nonsteroidal anti-inflammatory drugs. Lancet 1987;2:711-14.

6 Bjarnason I, Price AB, Zanelli G, et al. Clinicopathological eatures of nonsteroidal antiinflammatory drug-induced small intestinal strictures. Gastroenterology 1988;94:1070-4.

7 Bjarnason I, Zanelli G, Smith T, et al. The pathogenesis and consequence of non steroidal anti-inflammatory drug induced small intestinal inflammation in man. Scand $\mathcal{F}$ Rheumatol Suppl 1987;64(suppl 69):55-62.

8 Bjarnason I, Fehilly B, Smethurst P, et al. Importance of local versus systemic effects of non-steroidal antiinflammatory drugs in increasing small intestinal permeability in man. Gut 1991;32:275-7.

9 Bjarnason I, Hayllar J, Macpherson AJ, et al. Side effects of nonsteroidal anti-inflammatory drugs on the small and large intestine in humans. Gastroenterology 1993;104:1832-47.

10 Somasundaram S, Hayllar H, Rafi S, et al. The biochemical basis of non-steroidal anti-inflammatory drug-induced damage to the gastrointestinal tract: a review and a hypothesis. Scand F Gastroenterol 1995;30:289-99.

1 Mahmud T, Rafi SS, Scott DL, et al. Nonsteroidal antiinflammatory drugs and uncoupling of mitochondrial oxidative phosphorylation. Arthritis Rheum 1996;39:19982003.

12 Bjarnason I, Smethurst P, Fenn CG, et al. Misoprostol reduces indomethacin-induced changes in human small intestinal permeability. Dig Dis Sci 1989;34:407-11.

13 Bjarnason I, Williams P, Smethurst P, et al. Effect of non-steroidal anti-inflammatory drugs and prostaglandins on the permeability of the human small intestine. Gut on the permeabilit

14 Meade EA, Smith WL, DeWitt DL. Differential inhibition of prostaglandin endoperoxide synthase (cyclooxygenase) 
isozymes by aspirin and other non-steroidal antiinflammatory drugs. F Biol Chem 1993;268:6610-14

15 Fosslien E. Adverse effects of nonsteroidal antiinflammatory drugs on the gastrointestinal system. Ann Clin Lab Sci 1998;28:67-81.

16 Mitchell JA, Akarasereenont P, Thiemermann C, et al. Selectivity of nonsteroidal antiinflammatory drugs as inhibitors of constitutive and inducible cyclooxygenase. Proc Natl Acad Sci USA 1993;90:11693-7.

17 Ehrich E, Schnitzer T, Kivitz A, et al. MK-966, a highly selective COX-2 inhibitor, was effective in the treatment of osteoarthritis $(\mathrm{OA})$ of the knee and hip in a 6-week placebo controlled study. Arthritis Rheum 1999;40:S85.

18 Saag K, Fisher C, McKay J, et al. MK-0966, a specific COX-2 inhibitor has clinical efficacy comparable to ibuprofen in the treatment of knee and hip osteoarthritis (OA) in a 6-week controlled clinical trial. Arthritis Rheum 1998;41:S196.

19 Warner TD, Giuliano F, Vojnovic I, et al. Nonsteroid drug selectivities for cyclo-oxygenase-1 rather than cycloselectivities for cyclo-oxygenase-1 rather than cyclooxygenase-2 are associated with human gastrointestinal toxicity: a full in

20 Chan CC, Boyce S, Brideau C, et al. Rofecoxib [Vioxx, MK-0966; 4-(4'-methylsulfonylphenyl)-3-phenyl-2-(5H) furanone]: a potent and orally active cyclooxygenase- 2 inhibitor. Pharmacological and biochemical profiles. $f$ Pharmacol Exp Ther 1999;290:551-60.

21 Ehrich EW, Dallob A, De L I, et al. Characterization of rofecoxib as a cyclooxygenase- 2 isoform inhibitor and demonstration of analgesia in the dental pain model. Clin Pharmacol Ther 1999;65:336-47.

22 Cryer B, Gottesdiener K, Gertz B, et al. Effects of a novel cyclooxygenase (COX)-2 inhibitor on gastric mucosal prostaglandin (PG) synthesis in healthy humans. $A m \mathcal{F}$ Gastroenterol 1998;91:1907.

23 Lanza FL, Simon T, Quan H, et al. Selective inhibition of cyclooxygenase-2 (COX-2) with MK-0966 (250 mg Q.D.) is associated with less gastroduodenal damage than aspirin (ASA) $650 \mathrm{mg}$ Q.I.D. or ibuprofen (IBU) $800 \mathrm{mg}$ TIID. Gastroenterology 1998;112:A194.
24 Hunt R, Bowen B, James C, et al. COX-2 specific inhibition with MK-0966 25 or $50 \mathrm{mg}$ Q.D. over 4 weeks does not increase fecal blood loss. A controlled study with placebo and ibuprofen $800 \mathrm{mg}$ T.I.D. Am $\mathcal{F}$ Gastroenterol 1998;93: 1671.

25 Bjarnason I, Macpherson A, Hollander D. Intestinal permeability: an overview. Gastroenterology 1995;108:1566-

26 Bjarnason I, Maxton D, Reynolds AP, et al. Comparison of four markers of intestinal permeability in control subjects and patients with coeliac disease. Scand f Gastroenterol 1994;29:630-9.

27 Kendall MJ, Nutter S, Hawkins CF. Xylose test: effect of aspirin and indomethacin. BMF 1971;1:533-6.

28 Dyer NH, Kendall MJ, Hawkins CF. Malabsorption in rheumatoid disease. Ann Rheum Dis 1971;30:626-30.

29 Menzies IS, Mount JN, Wheeler MJ. Quantitative estimation of clinically important monosaccharides in plasma by rapid thin layer chromatography. Ann Clin Biochem 1978;15:65-76.

30 Laine L, Hawkey C, Harper SE, et al. Effect of the COX-2 specific inhibitor (C-2SI), rofecoxib, on ulcer formation: A double-blind comparison with ibuprofen and placebo. Gastroenterology 1999;116:A229.

31 Lanza FL, Rack MF, Simon TJ. et al. Specific inhibition of cyclooxygenase-2 with MK-0966 is associated with less gastroduodenal damage than either aspirin or ibuprofen. Aliment Pharmacol Ther 1999;13:761-7.

32 Sigthorsson G, Tibble J, Hayllar J, et al. Intestinal permeability and inflammation in patients on NSAIDs. Gut 1998;43:506-11.

33 Bjarnason I, Smethurst P, Clark P, et al. Effect of prostaglandin on indomethacin-induced increased intestinal permeability in man. Scand $\mathcal{F}$ Gastroenterol 1989; 164(suppl 146):97-102.

34 Bjarnason I, Zanelli G, Smith T, et al. Nonsteroidal antiinflammatory drug-induced intestinal inflammation in humans. Gastroenterology 1987;93:480-9. 